TRANSACTIONS OF THE

AMERICAN MATHEMATICAL SOCIETY

Volume 357, Number 7, Pages 2665-2679

S 0002-9947(05)03863-8

Article electronically published on March 1, 2005

\title{
ON HOMEOMORPHISM GROUPS OF MENGER CONTINUA
}

\author{
JAN J. DIJKSTRA
}

\begin{abstract}
It is shown that the homeomorphism groups of the (generalized) Sierpiński carpet and the universal Menger continua are not zero-dimensional. These results were corollaries to a 1966 theorem of Brechner. New proofs were needed because we also show that Brechner's proof is inadequate. The method by which we obtain our results, the construction of closed imbeddings of complete Erdős space in the homeomorphism groups, is of independent interest.
\end{abstract}

\section{INTRODUCTION}

Let $X$ be a (Peano) continuum and let $G$ be a subgroup of $\mathcal{H}(X)$, the autohomeomorphism group of $X$ with the compact-open topology. Then $X$ is called locally setwise homogeneous under $G$ if there exists a basis $\mathcal{B}$ of connected open subsets of $X$ and a dense subset $D$ of $X$ such that for any $B \in \mathcal{B}$ and any two points $x$ and $y$ of $B \cap D$ there is an $h \in G$ that is supported on $B$ and with $h(x)=y$. The following statement is presented as Theorem 2.1 in Brechner [5]:

Claim 1. If $X$ is a continuum that is locally setwise homogeneous under a closed subgroup $G$ of $\mathcal{H}(X)$, then $\operatorname{dim} G \geq 1$.

The author thanks Jan van Mill for informing him that there is a problem with the proof of this statement. Specifically, van Mill found that the proof of [5] Lemma 2.4], a key step in the argument leading to Claim 1, is not convincing. In fact, we show by counterexample that Lemma 2.4 in [5] is invalid; see $\S 3$. We do not know whether Claim 1 is valid but we were able to recover, by a different method, the most important applications of Claim 1. The first application is that the homeomorphism group of the $n$-dimensional Sierpiński carpet $M_{n}^{n+1}$ for $n \neq 3$ is at least onedimensional; see $\S 5$. The second application is that the homeomorphism group of the $n$-dimensional universal Menger continuum $\mu^{n}$ is at least one-dimensional; see $\S 6$. The dimension of these homeomorphism groups is completely determined because Oversteegen and Tymchatyn [17] proved that it is at most one. Thirdly, [5. Theorem 3.3] states that if $M$ is a compact topological manifold with $\operatorname{dim} M \geq 2$ and $D$ is a countable dense subset, then the group of autohomeomorphisms of $M$ that map $D$ onto itself is at least one-dimensional. This result was proved in Dijkstra and van Mill [11 and the proof can also be found here in $\S 4$.

We obtain our results by imbedding (complete) Erdős space in the homeomorphism groups in question. This fact is in itself significant since the existence of

Received by the editors July 15, 2003.

2000 Mathematics Subject Classification. Primary 57S05.

Key words and phrases. Menger continuum, Sierpiński carpet, homeomorphism group, topological dimension, complete Erdős space.

(c)2005 American Mathematical Society 2665

Reverts to public domain 28 years from publication 
these imbeddings forms a crucial step in the proofs of the results in Dijkstra and van Mill [10, 12, which give a complete topological classification of the groups of homeomorphisms of topological manifolds, of Menger manifolds, and of Hilbert cube manifolds that leave a countable dense subset invariant.

\section{Preliminaries}

All spaces are assumed to be separable metric.

For $m \in \mathbb{N}$ we will use the following metric on the topological vector space $\mathbb{R}^{m}$ : if $x=\left(x_{1}, \ldots, x_{m}\right) \in \mathbb{R}^{m}$ and $y=\left(y_{1}, \ldots, y_{m}\right) \in \mathbb{R}^{m}$, then $d(x, y)=\max _{i=1}^{m}\left|x_{i}-y_{i}\right|$. The zero vector is denoted by $\mathbf{0}$. If $A \subset \mathbb{R}^{m}$, then $\partial A$ denotes the boundary of $A$ in $\mathbb{R}^{m}$. An $m$-cell is any space that is homeomorphic to $\mathbb{I}^{m}$, where $\mathbb{I}=[0,1]$.

We shall use a number of compactifications of $\mathbb{R}^{m}$. Let $S^{m}$ denote the one-point compactification of $\mathbb{R}^{m}$. We let $\hat{\mathbb{R}}$ stand for the compactification $[-\infty, \infty]$ of $\mathbb{R}$. We shall use the convention $\pm \infty+t= \pm \infty$ when $t \in \mathbb{R}$. This allows us to extend the addition operation on $\mathbb{R}^{m}$ continuously to a function from $\hat{\mathbb{R}}^{m} \times \mathbb{R}^{m}$ to $\hat{\mathbb{R}}^{m}$. Let $\pi_{i}: \hat{\mathbb{R}}^{m} \rightarrow \hat{\mathbb{R}}$ be the coordinate projection given by $\pi_{i}(x)=x_{i}$. Finally, if $m \geq 2$, then we obtain a quotient space $Q^{m}$ from $\hat{\mathbb{R}}^{m}$ by identifying the faces $\left\{x \in \hat{\mathbb{R}}^{m}: x_{2}=\infty\right\}$ and $\left\{x \in \hat{\mathbb{R}}^{m}: x_{2}=-\infty\right\}$ to points $\alpha$, respectively $\beta$. Note that $Q^{m}$ is like $\hat{\mathbb{R}}^{m}$ an $m$-cell that compactifies $\mathbb{R}^{m}$. We let $\rho$ be a compatible metric on $Q^{m}$ such that $\rho(x, y) \leq d(x, y)$ for every $x, y \in \mathbb{R}^{m}$.

We will now recall the construction of the $n$-dimensional Menger continuum $M_{n}^{m}$ in $\mathbb{R}^{m}$ for $n, k \in \mathbb{N}$ with $n<m$. Start with the cube $C_{0}=\mathbb{I}^{m}$ and subdivide it into $3^{m}$ congruent subcubes. To get $C_{1}$ take the union of all the subcubes that meet the $n$-skeleton of $C_{0}$. Repeat the process on each of the cubes that make up $C_{1}$ and so on. We obtain a sequence $C_{0} \supset C_{1} \supset C_{2} \supset \cdots$ and we put $M_{n}^{m}=\bigcap_{i=0}^{\infty} C_{i}$. See Engelking [14 \$1.11] for details.

If $X$ is a locally compact space, then $\mathcal{H}(X)$ denotes the group of autohomeomorphisms of $X$ equipped with the compact-open topology. If $X$ is compact this topology coincides with the topology of uniform convergence and $\mathcal{H}(X)$ is a topological group. If every point in $X$ has a neighbourhood that is a continuum, then $\mathcal{H}(X)$ is a topological group as well since the compact-open topology coincides with the topology inherited from $\mathcal{H}(\alpha X)$, where $\alpha X$ is the one point compactification (see Dijkstra 8] and Arens [3]). If $O$ is an open subset of $X$, then $\mathcal{H}(X\lceil O)$ denotes the (closed) subgroup of $\mathcal{H}(X)$ that consists of homeomorphisms that are supported on $O$. If $A \subset X$, then $\mathcal{H}(X, A)$ denotes $\{h \in \mathcal{H}(X): h(A)=A\}$.

Let $\mathbb{Z}^{+}$stand for the set of nonnegative integers. Let $p \geq 1$ and consider the Banach space $\ell^{p}$. This space consists of all sequences $z=\left(z_{0}, z_{1}, z_{2}, \ldots\right)$ of real numbers such that $\sum_{i=0}^{\infty}\left|z_{i}\right|^{p}<\infty$. The topology on $\ell^{p}$ is generated by the norm $\|z\|=\left(\sum_{i=0}^{\infty}\left|z_{i}\right|^{p}\right)^{1 / p}$. It is well known that this topology is the weakest topology that makes all the coordinate projections $z \mapsto z_{i}$ and the norm function continuous. This fact can also be formulated as follows: the norm topology on $\ell^{p}$ is generated by the product topology (that is inherited from $\mathbb{R}^{\mathbb{Z}^{+}}$) together with the sets $\{z \in$ $\left.\ell^{2}:\|z\|<t\right\}$ for $t>0$. We extend the $p$-norm over $\mathbb{R}^{\mathbb{Z}^{+}}$by putting $\|z\|=\infty$ for each $z \in \mathbb{R}^{\mathbb{Z}^{+}} \backslash \ell^{p}$.

Now let $E_{0}, E_{1}, E_{2}, \ldots$ be a fixed sequence of subsets of $\mathbb{R}$ and let

$$
\mathcal{E}=\left\{z \in \ell^{p}: z_{n} \in E_{n} \text { for every } n \in \mathbb{Z}^{+}\right\}
$$


be a corresponding subspace of $\ell^{p}$. If we choose $p=2$ and $E_{n}=\mathbb{Q}$ for every $n$, then $\mathcal{E}$ is called Erdös space, and if $E_{n}=\mathbb{R} \backslash \mathbb{Q}$, then we obtain complete Erdös space; cf. Erdös [15] and Kawamura, Oversteegen, and Tymchatyn [16].

Recall that if $A_{0}, A_{1}, \ldots$ is a sequence of subsets of a space $X$, then

$$
\limsup _{n \rightarrow \infty} A_{n}=\bigcap_{n=0}^{\infty} \overline{\bigcup_{k=n}^{\infty} A_{k}} .
$$

For the spaces $\mathcal{E}$ we found the following results in [9]:

Proposition 1. If 0 is a cluster point of $\lim \sup _{n \rightarrow \infty} E_{n}$, then every nonempty clopen subset of $\mathcal{E}$ is unbounded (and hence $\operatorname{dim} \mathcal{E} \neq 0$ ).

Theorem 2. If every $E_{i}$ is closed in $\mathbb{R}$, then $\mathcal{E}$ is homeomorphic to complete Erdös space if and only if $\operatorname{dim} \mathcal{E}>0$ and every $E_{n}$ is zero-dimensional.

A space is called almost zero-dimensional if every point has a neighbourhood basis consisting of sets that are intersections of clopen sets of the space. If every $E_{i}$ is zero-dimensional, then the resulting space $\mathcal{E}$ is almost zero-dimensional. The reason lies in the well-known fact that closed balls in $\ell^{p}$ are also closed subsets of $\mathbb{R}^{\mathbb{Z}^{+}}$with the product topology. Oversteegen and Tymchatyn [17] have shown that every $\mathcal{H}\left(M_{n}^{m}\right)$ is almost zero-dimensional and that every almost zero-dimensional space is at most one-dimensional.

\section{A counterexample}

The following definitions were taken from Brechner [5]. A chain of sets $\mathcal{U}$ : $U_{1}, \ldots, U_{n}$ is a finite, ordered collection of subsets of a space $X$ such that $U_{i} \cap U_{j} \neq \emptyset$ iff $\bar{U}_{i} \cap \bar{U}_{j} \neq \emptyset$ iff $|i-j| \leq 1$ and $U_{i} \not \subset U_{j}$ for any pair $i \neq j$. A chain set for $\mathcal{U}$ is a set $\left\{a_{0}, \ldots, a_{n}\right\}$ such that $a_{0} \in U_{1} \backslash U_{2}, a_{i} \in U_{i} \cap U_{i+1}$ for $1 \leq i<n$, and $a_{n} \in U_{n} \backslash U_{n-1}$. A homeomorphism $H: X \rightarrow X$ is called a pseudo translation of $k$ units of $\mathcal{U}: U_{1}, \ldots, U_{n}$ if $H$ is supported on $\bigcup_{i=1}^{n} U_{n}$, if there exists a chain set $\left\{a_{0}, \ldots, a_{n}\right\}$ for $\mathcal{U}$ with $H\left(a_{i}\right)=a_{i+k}$ for $0 \leq i \leq n-k$ and $H\left(a_{i}\right) \in U_{n}$ for $n-k<i \leq n$, and if for each $i \in\{1, \ldots, n\}$

$$
H\left(\bar{U}_{i}\right) \subset \bigcup_{j=i-1}^{i+k} \bar{U}_{j} \backslash \bar{U}_{i-2},
$$

where we put $U_{i}=\emptyset$ if $i<1$ or $i>n$.

The following statement appears as Lemma 2.4 in Brechner [5].

Claim 2. Let $X$ be a locally setwise homogeneous continuum, let $\mathcal{U}: U_{1}, \ldots, U_{n}$ be a chain of connected open sets of $X$, and let $H$ be a pseudo translation of $k$ units on $\mathcal{U}$. Then there exists a chain $\mathcal{D}: D_{1}, \ldots, D_{n-k}$ of connected open sets of $X$ such that $D_{i} \subset U_{i}$ and $H\left(D_{i}\right) \subset U_{i+k}$ for $i=1, \ldots, n-k$.

Note that the claim is trivially valid if $n-k \leq 2$. For all other cases we present a counterexample.

Example 1. Assume that $n-k \geq 3$. Let $X$ be the 2 -cell $\hat{\mathbb{R}} \times \hat{\mathbb{R}}$. Select an $\varepsilon \in\left(0, \frac{1}{5}\right)$. For every $i \in \mathbb{Z}$ we define the open set $O_{i}=(i-1-\varepsilon, i+\varepsilon) \times \hat{\mathbb{R}}$ and the point 
$a_{i}=(i, 0) \in O_{i} \cap O_{i+1}$. We put

$$
\begin{aligned}
U_{1} & =(\{-\infty\} \times \hat{\mathbb{R}}) \cup \bigcup_{i \leq 1} O_{i}=[-\infty, 1+\varepsilon) \times \hat{\mathbb{R}}, \\
U_{i} & =O_{i} \quad \text { if } 1<i<n, \\
U_{n} & =(\{\infty\} \times \hat{\mathbb{R}}) \cup \bigcup_{i \geq n} O_{i}=(n-1-\varepsilon, \infty] \times \hat{\mathbb{R}} .
\end{aligned}
$$

Note that $\mathcal{U}: U_{1}, \ldots, U_{n}$ is a chain of connected open sets that covers $X$ and that $\left\{a_{0}, \ldots, a_{n}\right\}$ is a chain set for $\mathcal{U}$.

We now construct a pseudo translation $H$ on the chain. Select three continuous functions $\alpha, \beta, \gamma: \hat{\mathbb{R}} \rightarrow \mathbb{R}$ such that

$$
\begin{aligned}
& \alpha(\hat{\mathbb{R}}) \subset[0, k], \quad \alpha(0)=k, \quad \alpha(y)=0 \text { if }|y| \geq 1 ; \\
& \beta(\hat{\mathbb{R}}) \subset[0,2], \quad \beta(1+k+2 \varepsilon)=2, \quad \beta(x)=0 \text { if }|x-1-k-2 \varepsilon| \geq \varepsilon ; \\
& \gamma(\hat{\mathbb{R}}) \subset[-3 \varepsilon, 0], \quad \gamma(0)=0, \quad \gamma(y)=-3 \varepsilon \text { if }|y| \geq 1 .
\end{aligned}
$$

We define three homeomorphisms of $X$ as follows:

$$
\begin{aligned}
& h_{1}(x, y)=(x+\alpha(y), y), \\
& h_{2}(x, y)=(x, y+\beta(x)), \\
& h_{3}(x, y)=(x+\gamma(y), y),
\end{aligned}
$$

and we put $H=h_{3} \circ h_{2} \circ h_{1}$. Note that if $(x, y) \in X$, then $\pi_{1}\left(h_{1}(x, y)\right) \in[x, x+k]$, $\pi_{1}\left(h_{2}(x, y)\right)=x, \pi_{1}\left(h_{3}(x, y)\right) \in[x-3 \varepsilon, x]$, and hence $\pi_{1}(H(x, y)) \in[x-3 \varepsilon, x+k]$. For $i \in \mathbb{Z}$ we have $\bar{O}_{i}=[i-1-\varepsilon, i+\varepsilon] \times \hat{\mathbb{R}}$ and hence

$$
H\left(\bar{O}_{i}\right) \subset[i-1-4 \varepsilon, i+k+\varepsilon] \times \hat{\mathbb{R}} \subset \bigcup_{j=i-1}^{i+k} \bar{O}_{j} \backslash \bar{O}_{i-2}
$$

because $5 \varepsilon<1$. Consequently, $H$ satisfies condition $(*)$. If $i \in \mathbb{Z}$, then

$$
H\left(a_{i}\right)=h_{3} \circ h_{2} \circ h_{1}(i, 0)=h_{3} \circ h_{2}(i+k, 0)=h_{3}(i+k, 0)=(i+k, 0)=a_{i+k} .
$$

Assume now that $D_{1}, D_{2}, D_{3}$ is a chain of connected sets such that $D_{i} \subset U_{i}$ and $H\left(D_{i}\right) \subset U_{i+k}$ for $i=1,2,3$ (here we use that $n-k \geq 3$ ). We distinguish two cases.

Case I: $\pi_{2}\left(D_{2}\right) \not \subset(-1,1)$. Let $(x, y) \in D_{2}$ such that $y \notin(-1,1)$ and thus $1-\varepsilon<x<2+\varepsilon$. Observe that

$$
H(x, y)=h_{3} \circ h_{2} \circ h_{1}(x, y)=h_{3} \circ h_{2}(x, y)=h_{3}(x, y)=(x-3 \varepsilon, y) .
$$

Since $x-3 \varepsilon<2-2 \varepsilon$ we have that $H(x, y) \notin U_{2+k}$ which contradicts the assumption $H\left(D_{2}\right) \subset U_{2+k}$.

Case II: $\pi_{2}\left(D_{2}\right) \subset(-1,1)$. Select $\left(x_{1}, y_{1}\right) \in D_{1} \cap D_{2}$ and $\left(x_{2}, y_{2}\right) \in D_{2} \cap D_{3}$. So $\left(x_{1}, y_{1}\right) \in U_{1}$ and hence $x_{1}<1+\varepsilon$ and

$$
\pi_{1} \circ h_{1}\left(x_{1}, y_{1}\right)<1+k+\varepsilon .
$$

On the other hand, $H\left(x_{2}, y_{2}\right) \in H\left(D_{3}\right) \subset U_{3+k}$ which means that $\pi_{1} \circ H\left(x_{2}, y_{2}\right)>$ $2+k-\varepsilon$. Since $h_{3}$ is a shift to the left we have

$$
\pi_{1} \circ h_{1}\left(x_{2}, y_{2}\right)=\pi_{1} \circ h_{2} \circ h_{1}\left(x_{2}, y_{2}\right) \geq \pi_{1} \circ H(x, y)>2+k-\varepsilon>1+k+2 \varepsilon .
$$


Since $\pi_{1}\left(h_{1}\left(D_{2}\right)\right)$ is connected we can find a point $(x, y) \in D_{2}$ with $\pi_{1} \circ h_{1}(x, y)=$ $1+k+2 \varepsilon$. Observe that

$$
\begin{aligned}
H(x, y) & =h_{3} \circ h_{2} \circ h_{1}(x, y)=h_{3} \circ h_{2}(1+k+2 \varepsilon, y) \\
& =h_{3}(1+k+2 \varepsilon, y+2)=(1+k-\varepsilon, y+2) \\
& \notin U_{2+k}
\end{aligned}
$$

because $\pi_{2}\left(D_{2}\right) \subset(-1,1)$ and hence $y+2>1$. Again we have a contradiction with the assumption $H\left(D_{2}\right) \subset U_{2+k}$.

\section{Manifolds With an invariant Countable Dense Subset}

In this section we prove [5, Theorem 3.3], which was obtained in that paper as a corollary to the proof of Claim 1 The proof given here can also be found in [11. We have included it here for the sake of completeness and with the expectation that displaying the Erdős space imbedding idea in a relatively simple setting first will make the proofs in $\S 5$ and $\S 6$ more accessible.

Theorem 3. If $D$ is a countable dense subset of a topological manifold $M$ with $\operatorname{dim} M \geq 2$, then $\operatorname{dim} \mathcal{H}(M, D)=1$.

Proof. We define

$$
\mathcal{E}=\left\{z \in \ell^{1}: z_{i} \in \mathbb{Q}^{+} \text {for every } i \in \mathbb{Z}^{+}\right\},
$$

where $\mathbb{Q}^{+}=\mathbb{Q} \cap[0, \infty)$. Note that for $z \in \mathcal{E}$ we have $\|z\|=\sum_{i=0}^{\infty} z_{i}$. According to Proposition 1 we have $\operatorname{dim} \mathcal{E}>0$. Since it is shown in Dijkstra and van Mill [11] that $\mathcal{H}(M, D)$ is almost zero-dimensional it suffices to imbed $\mathcal{1} \mathcal{E}$ in $\mathcal{H}(M, D)$.

We first show that the statement is true for a 2-cell. We represent the 2-cell by $\hat{\mathbb{R}}^{2}$ and we let $\partial \hat{\mathbb{R}}^{2}$ denote the geometric boundary of $\hat{\mathbb{R}}^{2}$. We will imbed $\mathcal{E}$ in $\mathcal{H}\left(\hat{\mathbb{R}}^{2}, D\right) \cap \mathcal{H}\left(\hat{\mathbb{R}}^{2}\left\lceil\mathbb{R}^{2}\right)\right.$ which means that we can ignore any points of $D$ that fall on the geometric boundary. By countable dense homogeneity we may now assume that $D=\mathbb{Q}^{2}$. Let $b$ be an irrational number and select two sequences $p_{0}<p_{1}<\cdots$ and $q_{0}>q_{1}>\cdots$ of rational numbers that both converge to $b$. For every $z \in \mathcal{E}$ we define the function $\alpha_{z}: \hat{\mathbb{R}} \rightarrow \mathbb{R}$ by

$$
\alpha_{z}(x)= \begin{cases}0, & \text { if } x \in\left[-\infty, p_{0}\right] \cup\left[q_{0}, \infty\right] \\ \frac{z_{i}}{p_{i+1}-p_{i}}\left(x-p_{i}\right)+\sum_{k=0}^{i-1} z_{k}, & \text { if } p_{i} \leq x \leq p_{i+1} \text { for some } i \\ \frac{z_{i}}{q_{i+1}-q_{i}}\left(x-q_{i}\right)+\sum_{k=0}^{i-1} z_{k}, & \text { if } q_{i+1} \leq x \leq q_{i} \text { for some } i \\ \|z\|, & \text { if } x=b .\end{cases}
$$

Note that $\alpha_{z}\left(p_{i}\right)=\alpha_{z}\left(q_{i}\right)=\sum_{k=0}^{i-1} z_{i}$ for $z \in \mathcal{E}$ and $i \in \mathbb{Z}^{+}$and that $\alpha_{z}$ simply connects these points with linear segments. It is clear that $\alpha_{z}$ is well defined and continuous and that

$$
\left|\alpha_{z}(x)-\alpha_{z^{\prime}}(x)\right| \leq\left\|z-z^{\prime}\right\|
$$

for every $z, z^{\prime} \in \mathcal{E}$ and $x \in \hat{\mathbb{R}}$. Furthermore, since $\mathbb{Q}$ is a field we have that $\alpha_{z}(x) \in \mathbb{Q}$ whenever $x \in \mathbb{Q}$.

\footnotetext{
${ }^{1}$ It is proved in [10, 12] that $\mathcal{H}(M, D)$ is in fact homeomorphic to $\mathcal{E}$. The imbedding presented here plays an important role in that proof.
} 
For each $z \in \mathcal{E}$ we define the map $H_{z}: \hat{\mathbb{R}}^{2} \rightarrow \hat{\mathbb{R}}^{2}$ by $H(z)(x, y)=\left(x, y+\alpha_{z}(x)\right)$. Since $\alpha_{z}$ is bounded (by $\|z\|$ ), it is clear that $H_{z}$ is well defined and an element of $\mathcal{H}\left(\hat{\mathbb{R}}^{2}\right)$. Note also that every $H_{z}$ is supported on $\mathbb{R}^{2}$. Observe that for $(x, y) \in \mathbb{R}^{2}$,

$$
d\left(H_{z}(x, y), H_{z^{\prime}}(x, y)\right)=\left|\alpha_{z}(x)-\alpha_{z^{\prime}}(x)\right| \leq\left\|z-z^{\prime}\right\| .
$$

This means that $H_{z} \mid \mathbb{R}^{2}$ depends continuously on $z$ if we use the topology of uniform convergence on $\mathcal{H}\left(\mathbb{R}^{2}\right)$. So we certainly have that $H: \mathcal{E} \rightarrow \mathcal{H}\left(\hat{\mathbb{R}}^{2}\left\lceil\mathbb{R}^{2}\right)\right.$ is continuous. Observe that for each $z \in \mathcal{E}, i \in \mathbb{Z}^{+}$,

$$
H_{z}(b, 0)=(b,\|z\|) \quad \text { and } \quad H_{z}\left(p_{i}, 0\right)=\left(p_{i}, \sum_{k=0}^{i-1} z_{i}\right)
$$

and hence $\pi_{2}\left(H_{z}(b, 0)\right)=\|z\|$ and $\pi_{2}\left(H_{z}\left(p_{i+1}, 0\right)\right)-\pi_{2}\left(H_{z}\left(p_{i}, 0\right)\right)=z_{i}$. This means that $H$ is a one-to-one map. Since the norm topology is the weakest topology on $\ell^{1}$ that makes the coordinate projections and the norm function continuous, it follows that if we use $H$ to pull the topology of point-wise convergence on $\mathcal{H}\left(\hat{\mathbb{R}}^{2}\right)$ back to $\mathcal{E}$, then we get a topology that is at least as strong as the norm topology. Since we already know that $H$ is continuous and since the topology of uniform convergence is stronger than the topology of point-wise convergence, we have that $H$ is an imbedding. If $x, y \in \mathbb{Q}$ and $z \in E$, then $\alpha_{z}(x) \in \mathbb{Q}$ so $H_{z}(x, y)=\left(x, y+\alpha_{z}(x)\right) \in \mathbb{Q}^{2}$. If, on the other hand $H_{z}(x, y) \in \mathbb{Q}^{2}$, then $x \in \mathbb{Q}$ and thus $y \in \mathbb{Q}-\alpha_{z}(x)=\mathbb{Q}$. So $H$ is an imbedding of $\mathcal{E}$ in $\mathcal{H}\left(\hat{\mathbb{R}}^{2}, \mathbb{Q}^{2}\right) \cap \mathcal{H}\left(\hat{\mathbb{R}}^{2}\left\lceil\mathbb{R}^{2}\right)\right.$.

If $X$ is compact we obtain the suspension $S(X)$ by identifying the faces $X \times\{-\infty\}$ and $X \times\{\infty\}$ to points in the product $X \times \hat{\mathbb{R}}$. Assume now that $G=\mathcal{H}\left(\hat{\mathbb{R}}^{n}, \mathbb{Q}^{n}\right) \cap$ $\mathcal{H}\left(\hat{\mathbb{R}}^{n}\left\lceil\mathbb{R}^{n}\right)\right.$ contains a copy of $\mathcal{E}$ for some $n \geq 2$. We then have that $S\left(\hat{\mathbb{R}}^{n}\right)$ is an $(n+1)$-cell and that $S\left(\partial \hat{\mathbb{R}}^{n}\right)$ is its geometric boundary. We can imbed $G$ as a closed subgroup of $\mathcal{H}\left(S\left(\hat{\mathbb{R}}^{n}\right), \mathbb{Q}^{n+1}\right) \cap \mathcal{H}\left(S\left(\hat{\mathbb{R}}^{n}\right)\left\lceil\mathbb{R}^{n+1}\right)\right.$ by multiplying with the identity on $\hat{\mathbb{R}}$. So we may conclude by induction that for every $n \geq 2$ and countable dense set $D \subset \mathbb{I}^{n}$ the group $\mathcal{H}\left(\mathbb{I}^{n}, D\right) \cap \mathcal{H}\left(\mathbb{I}^{n}\left\lceil(0,1)^{n}\right)\right.$ contains a copy of $\mathcal{E}$. Obviously, this group can be imbedded into $\mathcal{H}\left(M, D^{\prime}\right)$ for every $n$-dimensional manifold $M$ and countable dense subset $D^{\prime}$.

Remark 1 . Note that $\mathcal{E}$ is a semigroup with respect to addition. Clearly, $\alpha_{z+z^{\prime}}=$ $\alpha_{z}+\alpha_{z^{\prime}}$ for $z, z^{\prime} \in \mathcal{E}$ and we have that $H$ is a semigroup homomorphism. We can extend $H$ to a continuous monomorphism over the group $G=\ell^{1} \cap \mathbb{Q}^{\mathbb{Z}^{+}}$, but then the topology on $H(G)$ is strictly weaker than the norm topology on $G$. We now verify that $H: \mathcal{E} \rightarrow \mathcal{H}\left(\hat{\mathbb{R}}^{2}, \mathbb{Q}^{2}\right)$ is a closed imbedding and hence that every $\mathcal{H}(M, D)$ as in the theorem contains a closed copy of $\mathcal{E}$. Let $h \in \mathcal{H}\left(\hat{\mathbb{R}}^{2}, \mathbb{Q}^{2}\right)$ be such that there is a sequence $z^{1}, z^{2}, \ldots$ in $\mathcal{E}$ with $\lim _{j \rightarrow \infty} H_{z^{j}}=h$. Observe that since $h\left(\mathbb{Q}^{2}\right)=\mathbb{Q}^{2}$ we have that

$$
\begin{aligned}
z_{i} & =\pi_{2}\left(h\left(p_{i+1}, 0\right)\right)-\pi_{2}\left(h\left(p_{i}, 0\right)\right) \\
& =\lim _{j \rightarrow \infty}\left(\pi_{2}\left(H_{z^{j}}\left(p_{i+1}, 0\right)\right)-\pi_{2}\left(H_{z^{j}}\left(p_{i}, 0\right)\right)\right) \\
& =\lim _{j \rightarrow \infty} z_{i}^{j}
\end{aligned}
$$

is well defined and an element of $\mathbb{Q}^{+}$for each $i \in \mathbb{Z}^{+}$. Since $\mathcal{H}\left(\hat{\mathbb{R}}^{2}\left\lceil\mathbb{R}^{2}\right)\right.$ is closed we have $h\left(\mathbb{R}^{2}\right)=\mathbb{R}^{2}$ and hence $\pi_{2}(h(b, 0))<\infty$. We have $\pi_{2}\left(h\left(p_{i}, 0\right)\right)=\sum_{k=0}^{i-1} z_{k}$ 
which means that

$$
\lim _{j \rightarrow \infty}\left\|z^{j}\right\|=\lim _{j \rightarrow \infty} \pi_{2}\left(H_{z^{j}}(b, 0)\right)=\pi_{2}(h(b, 0))=\|z\|<\infty .
$$

So $z=\left(z_{0}, z_{1}, \ldots\right) \in \mathcal{E}$ and $\lim _{j \rightarrow \infty} z^{j}=z$ because the topology on $\mathcal{E}$ is generated by the coordinate projections in conjunction with the norm. In conclusion, $h=$ $\lim _{j \rightarrow \infty} H_{z^{j}}=H_{z}$.

\section{SiERPIŃSKI CARPETS}

Let $n \in \mathbb{N}$. A nowhere dense compact subset $X$ of $S^{n+1}$ is called an $n$ dimensional Sierpinski carpet if the collection of components $\left\{U_{i}: i \in \mathbb{N}\right\}$ of $S^{n+1} \backslash$ $X$ forms a null sequence such that the closures of the $U_{i}$ 's are a pairwise disjoint collection and every $S^{n+1} \backslash U_{i}$ is an $(n+1)$-cell. The following characterization theorem is due to Whyburn [18] (for $n=1$ ) and Cannon [7] (for $n \geq 2$ ).

Theorem 4. Let $X$ and $Y$ be two n-dimensional Sierpinski carpets for $n \in \mathbb{N} \backslash$ $\{3\}$ and let $U$ and $V$ be components of $S^{n+1} \backslash X$, respectively $S^{n+1} \backslash Y$. If $h$ is a homeomorphism from the boundary of $U$ to the boundary of $V$, then $h$ can be extended to a homeomorphism from $X$ to $Y$.

The Menger continuum $M_{n}^{n+1}$ is a standard example of an $n$-dimensional Sierpiński carpet.

Theorem 5. If $n \in \mathbb{N} \backslash\{3\}$, then $\operatorname{dim} \mathcal{H}\left(M_{n}^{n+1}\right)=1$.

Proof. Consider the following closed subspace of $\ell^{1}$ :

$$
\mathcal{E}=\left\{z \in \ell^{1}: 2^{i} z_{i} \in \mathbb{Z}^{+} \text {for } i \in \mathbb{Z}^{+}\right\} .
$$

According to Proposition 1 we have $\operatorname{dim} \varepsilon \geq 1$. Since Oversteegen and Tymchatyn 17] proved that $\operatorname{dim} \mathcal{H}\left(M_{n}^{n+1}\right) \leq 1$ it suffices to show that $\mathcal{E}$ can be imbedded in $\mathcal{H}\left(M_{n}^{n+1}\right)$. Let $u=(1,0, \ldots, 0)$ and $v=(0,1,0, \ldots, 0)$ be unit vectors in $\mathbb{R}^{n+1}$. We will construct a topological copy $\bar{B}$ of $M_{n}^{n+1}$ that contains the set $\{\mathbf{0}\} \bigcup\left\{2^{-i+1} u: i \in\right.$ $\left.\mathbb{Z}^{+}\right\}$. We will then construct an imbedding $\bar{H}: \mathcal{E} \rightarrow \mathcal{H}(\bar{B})$ such that for each $z \in \mathcal{E}$ we have

$$
\bar{H}(z)(\mathbf{0})=\|z\| v \quad \text { and } \quad \bar{H}(z)\left(2^{-i+1} u\right)=2^{-i+1} u+\sum_{k=0}^{i-1} z_{k} v
$$

for every $i \in \mathbb{Z}^{+}$. Note the analogy with the construction in $\S 4$. We will now concentrate on constructing a "shear transformation" $\sigma$ that can be used to connect the vertical shifts that occur at at the points $2^{-i+1} u$ with each other. In $\S 4$ similar shifts occurred at the points $\left(p_{i}, 0\right)$, but there it was possible to simply connect them with straight lines.

Consider the unit cube $C=[1,2] \times \mathbb{I}^{n}$ in $\mathbb{R}^{n+1}$. We split $C$ into two triangular cells:

$$
T_{1}=\left\{x \in C: x_{1}+x_{2} \leq 2\right\} \quad \text { and } \quad T_{2}=\left\{x \in C: x_{1}+x_{2} \geq 2\right\} .
$$

Note that $D=T_{2} \cup\left(T_{1}+v\right)$ is also an $(n+1)$-cell and that $\varphi: C \rightarrow D$ defined by $\varphi(x)=x+\left(2-x_{1}\right) v$ is a homeomorphism. Let $S_{1}$ and $S_{2}$ be $n$-dimensional Sierpiński carpets that are obtained by deleting a suitable null sequence of open sets $U_{i}$ from the interiors of $T_{1}$, respectively $T_{2}$. Note that $K=S_{1} \cup S_{2}$ and $L=S_{2} \cup\left(S_{1}+v\right)$ are also $n$-dimensional Sierpiński carpets and that $\partial C$ and $\partial D$ are the boundaries of the unbounded components of $\mathbb{R}^{n+1} \backslash K$, respectively $\mathbb{R}^{n+1} \backslash L$. According to 
Theorem 4 there is a homeomorphism $\sigma: K \rightarrow L$ that extends $\varphi\lceil\partial C$. Since both domain and range of $\sigma$ are contained in $[1,2] \times[0,2] \times \mathbb{I}^{n-1}$ we have $d(\sigma(x), x) \leq 2$ for every $x \in K$. We define the closed subsets $N$ and $F$ of $\mathbb{R}^{n+1}$ as follows:

$$
N=C+\{k v: k \in \mathbb{Z}\}=D+\{k v: k \in \mathbb{Z}\}=[1,2] \times \mathbb{R} \times \mathbb{I}^{n-1}
$$

and

$$
F=K+\{k v: k \in \mathbb{Z}\}=L+\{k v: k \in \mathbb{Z}\} \subset N .
$$

Let $x \in(K-v) \cap K$ so $x_{2}=0$. Then $x \in \partial C$ and $x+v \in \partial C$ so $\sigma(x+v)=$ $x+v+\left(2-x_{1}\right) v=\sigma(x)+v$. We also have $\sigma((K-v) \cap K)=(L-v) \cap L$. These observations mean that we can extend $\sigma$ to an autohomeomorphism of $F$ by the rule $\sigma(x+k v)=\sigma(x)+k v$ for $x \in K$ and $k \in \mathbb{Z}$. Note that if $x \in F$ and $x_{1}=2$, then $\sigma(x)=\varphi(x)=x$ and that if $x_{1}=1$, then $\sigma(x)=\varphi(x)=x+v$. Thus the map $\sigma$ is a shear transformation that connects the identity on the "right face" of $F$ with a vertical shift by 1 unit on the "left face" of $F$. In particular, $\sigma(2 u)=2 u$ and $\sigma(u)=u+v$. Note also that $F+k v=F$ for each $k \in \mathbb{Z}$.

Let $i \in \mathbb{Z}^{+}$. We define reduced copies of $L$ and $F$ :

$$
N_{i}=2^{-i} L \quad \text { and } \quad F_{i}=2^{-i} F .
$$

We also define $\sigma_{i}: F_{i} \rightarrow F_{i}$ by

$$
\sigma_{i}\left(2^{-i} x\right)=2^{-i} \sigma(x) \text { for } x \in F .
$$

Note that if $x \in F_{i}$, then $d\left(\sigma_{i}(x), x\right) \leq 2 \cdot 2^{-i}$. We have that $\pi_{1}\left(N_{i}\right)=\pi_{1}\left(F_{i}\right)=$ $\left[2^{-i}, 2^{-i+1}\right]$. For elements of the "left and right faces" of $F_{i}$ we have: $\sigma_{i}(x)=x$ whenever $x_{1}=2^{-i+1}$, and $\sigma_{i}(x)=x+2^{-i} v$ whenever $x_{1}=2^{-i}$. We also have that $F_{i}+2^{-i} k v=F_{i}$ for every $k \in \mathbb{Z}$.

For $i \in \mathbb{Z}^{+}$and $z \in E$ we define the following autohomeomorphism $H_{i}(z)$ of $F_{i}$ : when $x \in F_{i}$, then

$$
H_{i}(z)(x)=\left(\sigma_{i}\right)^{j}(x)+\sum_{k=0}^{i-1} z_{k} v, \quad \text { where } j=2^{i} z_{i}
$$

while noting that $2^{i} z_{i} \in \mathbb{Z}^{+}$and that $\sum_{k=0}^{i-1} z_{k}$ is an element of $2^{-i} \mathbb{Z}$. Observe that

$$
H_{i}\left(2^{-i+1} u\right)=2^{-i+1} u+\sum_{k=0}^{i-1} z_{k} v \quad \text { and } \quad H_{i}\left(2^{-i} u\right)=2^{-i} u+\sum_{k=0}^{i} z_{k} v
$$

and that the shear transformation $\sigma_{i}$ is used to connect these two translations on opposite faces of $F_{i}$.

Let $z, z^{\prime} \in E$ and assume that, for instance, $j=2^{i} z_{i} \geq j^{\prime}=2^{i} z_{i}^{\prime}$. Consider for $x \in F_{i}$,

$$
\begin{aligned}
H_{i}(z)(x)-H_{i}\left(z^{\prime}\right)(x) & =\left(\sigma_{i}\right)^{j}(x)-\left(\sigma_{i}\right)^{j^{\prime}}(x)+\sum_{k=0}^{i-1}\left(z_{k}-z_{k}^{\prime}\right) v \\
& =\left(\sigma_{i}\right)^{j-j^{\prime}}\left(\left(\sigma_{i}\right)^{j^{\prime}}(x)\right)-\left(\sigma_{i}\right)^{j^{\prime}}(x)+\sum_{k=0}^{i-1}\left(z_{k}-z_{k}^{\prime}\right) v .
\end{aligned}
$$


Since $d\left(\sigma_{i}(y), y\right) \leq 2 \cdot 2^{-i}$ we have

$$
\begin{aligned}
d\left(H_{i}(z)(x), H_{i}\left(z^{\prime}\right)(x)\right) & \leq 2\left(j-j^{\prime}\right) 2^{-i}+\left|\sum_{k=0}^{i-1}\left(z_{k}-z_{k}^{\prime}\right)\right| \\
& \leq 2 \sum_{k=0}^{i}\left|z_{k}-z_{k}^{\prime}\right| \\
& \leq 2\left\|z-z^{\prime}\right\| .
\end{aligned}
$$

Moreover,

$$
d\left(H_{i}(z)(x), x+\sum_{k=0}^{i-1} z_{k} v\right) \leq d\left(\left(\sigma_{i}\right)^{j}(x), x\right) \leq 2 j 2^{-i}=2 z_{i} .
$$

We now take the following unions:

$$
A=\bigcup_{i=0}^{\infty} N_{i} \text { and } B=\bigcup_{i=0}^{\infty} F_{i}
$$

Let $V$ stand for the line $\mathbb{R} v=\{0\} \times \mathbb{R} \times\{0\} \times \cdots \times\{0\}$ in $\mathbb{R}^{n+1}$. We let $\bar{A}$ and $\bar{B}$ be the closures of $A$, respectively $B$, in $Q^{n+1}$. Note that $\bar{A}=A \cup V \cup\{\alpha, \beta\}$ and $\bar{B}=B \cup V \cup\{\alpha, \beta\}$. It is easily seen that $A_{0}=\left\{x \in \bar{A}: x_{2}=0\right\}$ is an $n$-cell, that $\bar{A}$ is the suspension of $A_{0}$ and hence an $(n+1)$-cell, and that Theorem 4 implies that $\bar{B}$ is homeomorphic to $M_{n}^{n+1}$.

For every $z \in E$ we define the autohomeomorphism $H(z)$ of $B$ by

$$
H(z)=\bigcup_{i=0}^{\infty} H_{i}(z)
$$

In order to verify that $H(z)$ is well defined we need to look at the overlaps between the $F_{i}^{j}$ 's. Since $\pi_{1}\left(F_{i}\right)=\left[2^{-i}, 2^{-i+1}\right]$ we only have to consider that case that $x \in F_{i} \cap F_{i+1}$ and $x_{1}=2^{-i}$. Put $j=2^{i} z_{i}$ and $j^{\prime}=2^{i+1} z_{i+1}$. Recall that $\sigma_{i}(x)=x+2^{-i} v$ and hence $\left(\sigma_{i}\right)^{j}(x)=x+j 2^{-i} v$. We now have

$$
H_{i}(z)(x)=\left(\sigma_{i}\right)^{j}(x)+\sum_{k=0}^{i-1} z_{k} v=x+j 2^{-i} v+\sum_{k=0}^{i-1} z_{k} v=x+\sum_{k=0}^{i} z_{k} v
$$

and, noting that $\sigma_{i+1}(x)=x$,

$$
H_{i+1}(z)(x)=\left(\sigma_{i+1}\right)^{j^{\prime}}(x)+\sum_{k=0}^{i} z_{k} v=x+\sum_{k=0}^{i} z_{k} v .
$$

Recall that we proved above that if $x \in B$ such that $2^{-i} \leq x_{1} \leq 2^{-i+1}$ for some $i \in \mathbb{Z}^{+}$, then $d\left(H(z)(x), x+\sum_{k=0}^{i-1} z_{k} v\right) \leq 2 z_{i}$. This result implies that we can extend $H(z)$ to a continuous map $\bar{H}(z)$ from $\bar{B}$ to $\bar{B}$ by defining

$$
\bar{H}(z)(x)= \begin{cases}H(z)(x), & \text { if } x \in B ; \\ x+\|z\| v, & \text { if } x \in V ; \\ \alpha, & \text { if } x=\alpha ; \\ \beta, & \text { if } x=\beta\end{cases}
$$


Since $\bar{H}(z)$ is obviously a bijection we have that $\bar{H}(z) \in \mathcal{H}(\bar{B})$ so $\bar{H}$ is a function from $\mathcal{E}$ to $\mathcal{H}(\bar{B})$. We proved earlier that

$$
\rho\left(H(z)(x), H\left(z^{\prime}\right)(x)\right) \leq d\left(H(z)(x), H\left(z^{\prime}\right)(x)\right) \leq 2\left\|z-z^{\prime}\right\|
$$

for any $z, z^{\prime} \in \mathcal{E}$ and $x \in B$. By density this means that $\rho\left(\bar{H}(z)(x), \bar{H}\left(\left(z^{\prime}\right)(x)\right) \leq\right.$ $2\left\|z-z^{\prime}\right\|$ for every $x \in \bar{B}$ and we have shown that $\bar{H}(z)$ depends continuously on $z$.

Let $p: Q^{n+1} \rightarrow \hat{\mathbb{R}}$ be the continuous function that is defined by

$$
p(x)= \begin{cases}x_{2}, & \text { if } x \in \hat{\mathbb{R}} \times \mathbb{R} \times \hat{\mathbb{R}}^{n-1} ; \\ \infty, & \text { if } x=\alpha ; \\ -\infty, & \text { if } x=\beta .\end{cases}
$$

Let $z \in \mathcal{E}$ and $i \in \mathbb{Z}^{+}$and recall that $\bar{H}(z)\left(2^{-i+1} u\right)=2^{-i+1} u+\sum_{k=0}^{i-1} z_{k} v=$ $\left(2^{-i+1}, \sum_{k=0}^{i-1} z_{k}, 0, \ldots, 0\right)$. Consequently, $p\left(\bar{H}(z)\left(2^{-i} u\right)\right)-p\left(\bar{H}(z)\left(2^{-i+1} u\right)\right)=z_{i}$ for each $i \in \mathbb{Z}^{+}$and hence $\bar{H}$ is one-to-one. Also note that $p(\bar{H}(z)(\mathbf{0}))=\|z\|$. Since the norm topology is generated by the coordinate projections in conjunction with the norm function, we have that $\bar{H}$ is an imbedding of $\mathcal{E}$ into $\mathcal{H}(\bar{B})$ by the same argument as we employed in $\S 4$.

Remark 2. According to Theorem 2 the space $\mathcal{E}$ is homeomorphic to complete Erdös space. Observe that $\mathcal{E}$ is a closed semigroup in $\ell^{1}$ with respect to addition. Since $\sigma_{i}\left(x+2^{-i} k v\right)=\sigma_{i}(x)+2^{-i} k v$ for $i \in \mathbb{Z}^{+}, x \in F_{i}$, and $k \in \mathbb{Z}$ we have that $H\left(z+z^{\prime}\right)=H(z) \circ H\left(z^{\prime}\right)$. Consequently, $\bar{H}$ is a semigroup homomorphism.

Remark 3 . We note a number of properties of the imbedding $\bar{H}$ that will be needed in a forthcoming paper of Dijkstra and van Mill [12. First, we observe that since every $H_{i}(z)(x)$ depends only on the coordinates $z_{0}, \ldots, z_{i}$ of $z$ for fixed $x$ we have that if $x \in \bar{B} \backslash V$, then $\bar{H}(z)(x)$ depends on only finitely many coordinates of $z \in \mathcal{E}$.

Let $Z$ stand for $\mathcal{E}$ but equipped with the zero-dimensional topology that $Z$ inherits from the product space $\mathbb{R}^{\mathbb{Z}^{+}}$, that is, the topology that is generated by the coordinate projections. Let $\psi: \mathcal{H}(\bar{B}) \rightarrow \bar{B}^{A}$ be defined by the rule $\psi(h)=h \uparrow A$, where $A=\left\{2^{-i+1} u: i \in \mathbb{Z}^{+}\right\} \cup\{\alpha\}$. We verify that $\psi \circ \bar{H}: Z \rightarrow \psi(\mathcal{H}(\bar{B}))$ is a closed imbedding. First we note that it is an imbedding because $\left.\bar{H}_{z}(\alpha)\right)=\alpha$ and $\bar{H}_{z}\left(2^{-i+1} u\right)=2^{-i+1} u+\sum_{k=0}^{i-1} z_{k} v$ for $z \in Z$ and $i \in \mathbb{Z}^{+}$. Let $h \in \mathcal{H}(\bar{B})$ be such that there is a sequence $z^{1}, z^{2}, \ldots$ in $Z$ with $\lim _{j \rightarrow \infty} \bar{H}\left(z^{j}\right) \uparrow A=h \uparrow A$ in $\bar{B}^{A}$. Since $\alpha \in A$ we have $h(\alpha)=\alpha$. The result is that for every $i \in \mathbb{Z}^{+}$,

$$
\begin{aligned}
z_{i} & =p\left(h\left(2^{-i} u\right)\right)-p\left(h\left(2^{-i+1} u\right)\right) \\
& =\lim _{j \rightarrow \infty}\left(p\left(\bar{H}\left(z^{j}\right)\left(2^{-i} u\right)\right)-p\left(\bar{H}\left(z^{j}\right)\left(2^{-i+1} u\right)\right)\right) \\
& =\lim _{j \rightarrow \infty} z_{i}^{j}
\end{aligned}
$$

is a well-defined real number. Since $z_{i}^{j} \in 2^{-i} \mathbb{Z}^{+}$and this set is closed in $\mathbb{R}$ we have that $z_{i} \in 2^{-i} \mathbb{Z}^{+}$for every $i \in \mathbb{Z}^{+}$. Note that $h(\mathbf{0})=\lim _{i \rightarrow \infty} h\left(2^{-i} u\right) \in[0, \infty) v$ because $h(\mathbf{0}) \neq h(\alpha)$. By the definition of the $z_{k}$ 's we have $p\left(h\left(2^{-i+1} u\right)\right)=\sum_{k=0}^{i-1} z_{k}$ which means that

$$
\|z\|=\lim _{i \rightarrow \infty} p\left(h\left(2^{-i+1} u\right)\right)=p\left(h\left(\lim _{i \rightarrow \infty} 2^{-i+1} u\right)\right)=p(h(\mathbf{0}))<\infty .
$$


Thus $z=\left(z_{0}, z_{1}, \ldots\right) \in Z$ and $\lim _{j \rightarrow \infty} z^{j}=z$ in $Z$. This means that $\psi(\bar{H}(z))=$ $\lim _{j \rightarrow \infty} \psi\left(\bar{H}\left(z^{j}\right)\right)=\psi(h)$ and the proof is finished

We now consider the imbedding $\bar{H}: \mathcal{E} \rightarrow \mathcal{H}(\bar{B})$ and verify that it is also a closed map. Let $h \in \mathcal{H}(\bar{B})$ be such that there is a sequence $z^{1}, z^{2}, \ldots$ in $\mathcal{E}$ with $\lim _{j \rightarrow \infty} \bar{H}\left(z^{j}\right)=h$. Then clearly $\lim _{j \rightarrow \infty} \bar{H}\left(z^{j}\right)\left\lceil A=h \uparrow A\right.$ in $\bar{B}^{A}$. By the preceding argument there is a $z \in \mathcal{E}$ that is the limit of $\left(z^{j}\right)_{j}$ in $Z$ and with the property $\|z\|=p(h(\mathbf{0}))=\lim _{j \rightarrow \infty} p\left(\bar{H}\left(z^{j}\right)(\mathbf{0})\right)=\lim _{j \rightarrow \infty}\left\|z^{j}\right\|$. Since the the topology on $\mathcal{E}$ is generated by the topology on $Z$ in conjunction with the norm function we have that $\lim _{j \rightarrow \infty} z^{j}=z$ in $\mathcal{E}$. Thus $h=\lim _{j \rightarrow \infty} \bar{H}\left(z^{j}\right)=\bar{H}(z)$.

Remark 4. If $O$ is a nonempty open subset of $M_{n}^{n+1}$ for $n \in \mathbb{N} \backslash\{3\}$, then the subgroup $\mathcal{H}\left(M_{n}^{n+1}\lceil O)\right.$ of $\mathcal{H}\left(M_{n}^{n+1}\right)$ consisting of homeomorphisms that are supported on $O$ is also one-dimensional. We mention this slight generalization because it is needed in Dijkstra, van Mill, and Steprāns [13. Consider the space $\mathcal{E}$ in the proof and the obviously homeomorphic subspace $\mathcal{E}^{\prime}=\left\{\left(0, z_{0}, z_{1}, \ldots\right): z \in \mathcal{E}\right\}$. Every $\bar{H}(z)$ for $z \in \mathcal{E}^{\prime}$ fixes $F_{0}^{0}=F$. Let $U$ be a "hole" of $F$, that is, a component of $N \backslash F$. Then $\mathcal{H}(\bar{B}\lceil\bar{B} \backslash \partial U)$ is one-dimensional because this group contains $\bar{H}\left(\mathcal{E}^{\prime}\right)$. According to Theorem 4 there is a homeomorphism $h: \bar{B} \rightarrow M_{n}^{n+1}$ that maps the boundary of $U$ onto $\partial I^{n+1}$ and hence $G=\mathcal{H}\left(M_{n}^{n+1}\left\lceil M_{n}^{n+1} \backslash \partial I^{n+1}\right)\right.$ is one-dimensional as well. Furthermore, because of the fractal nature of $M_{n}^{n+1}$ there is an imbedding $f$ of $M_{n}^{n+1}$ into $O$ such that $f\left(\partial I^{n+1}\right)$ contains the boundary of $M=f\left(M_{n}^{n+1}\right)$ in $M_{n}^{n+1}$. Let $U$ be the interior of $M$ in $M_{n}^{n+1}$. So $\mathcal{H}(M\lceil U)$ contains a copy of $G$ and is obviously identical to $\mathcal{H}\left(M_{n}^{n+1}\lceil U)\right.$, which in turn is contained in $\mathcal{H}\left(M_{n}^{n+1}\lceil O)\right.$.

Consider a null sequence $\left\{O_{i}: i \in \mathbb{N}\right\}$ of pairwise disjoint open subsets of $M_{n}^{n+1}$. Every $\mathcal{H}\left(M_{n}^{n+1}\left\lceil O_{i}\right)\right.$ contains a closed copy of $\mathcal{E}$ and hence $\mathcal{H}\left(M_{n}^{n+1}\left\lceil\bigcup_{i=1}^{\infty} O_{i}\right)\right.$ contains a closed copy of the product $\mathcal{E}^{\mathbb{N}}$. Every almost zero-dimensional complete space admits a closed imbedding into $\mathcal{E}^{\mathbb{N}}$, a property that distinguishes that space from $\mathcal{E}$; see Dijkstra, van Mill, and Steprāns [13]. Thus we have that every subset of $\mathcal{H}\left(M_{n}^{n+1}\right)$ with a nonempty interior contains a closed copy of every complete almost zero-dimensional space. Note that the spaces $\mathcal{H}(M, D)$ of Theorem 3 are also universal elements of the class of almost zero-dimensional spaces.

\section{Universal Menger continua}

In this section we prove that the homeomorphism groups of the universal Menger continua are one-dimensional. Our proof runs along the same lines as the proof of Theorem [5] and in our presentation we will focus on the differences. Particular attention is paid to how the Z-set Unknotting Theorem [4, 3.1.5] is used to produce the shear transformation $\sigma$.

Let $n \in \mathbb{Z}^{+}$. A space $X$ is called $n$-connected $\left(\mathrm{C}^{n}\right)$ if for every $k \in\{0, \ldots, n\}$ every map $f: \partial I^{k+1} \rightarrow X$ can be extended over $\mathbb{I}^{k+1}$. A space $X$ is called locally $n$-connected ( $\left.\mathrm{LC}^{n}\right)$ if for every $x \in X$ and every neighbourhood $U$ of $x$ there is a neighbourhood $V$ of $x$ such that for every $k \in\{0, \ldots, n\}$ every map $f: \partial I^{k+1} \rightarrow V$ can be extended over to a map from $\mathbb{I}^{k+1}$ to $U$. A metric space $X$ is said to have the disjoint $n$-disks property $\left(\mathrm{DD}^{n} \mathrm{P}\right)$ if for every $\varepsilon>0$ and maps $f, g: \mathbb{I}^{n} \rightarrow X$ there exist maps $f^{\prime}, g^{\prime}: \mathbb{I}^{n} \rightarrow X$ such that $f^{\prime}$ and $g^{\prime}$ are $\varepsilon$-close to $f$, respectively, $g$ and $f^{\prime}\left(\mathbb{I}^{n}\right) \cap g^{\prime}\left(\mathbb{I}^{n}\right)=\emptyset$. A closed subset $A$ of a compact metric space $X$ is called a $Z$-set if for every $\varepsilon>0$ there is a map $f: X \rightarrow X \backslash A$ that is $\varepsilon$-close to the identity. 
The following characterization of the universal Menger continuum $\mu^{n}$ is due to Bestvina [4, while the case $n=1$ is essentially due to Anderson [1, 2].

Theorem 6. A compact space $X$ is homeomorphic to $\mu^{n}$ if and only if $\operatorname{dim} X=n$, $X$ is $\mathrm{C}^{n-1}$ and $\mathrm{LC}^{n-1}$, and $X$ satisfies the $\mathrm{DD}^{n} \mathrm{P}$.

Remark 5. The space $M_{n}^{m}$ for $m \geq 2 n+1$ is a standard example of an $n$-dimensional universal Menger continuum; see Bestvina [4, p. 98]. This follows from the proof of the universality of $M_{n}^{m}$; cf. Engelking [14 $\left.\S 1.11\right]$. If $F$ is a $k$-face of $\mathbb{I}^{m}$, then we will call the intersection $F \cap M_{n}^{m}$ a $k$-face of $M_{n}^{m}$. Note that if $n<k<m$, then any $k$-face of $M_{n}^{m}$ is a copy of $M_{n}^{k}$. Let $F$ be an $(m-1)$-face of $M_{n}^{m}$, for instance $F=M_{n}^{m} \cap \pi_{1}^{-1}(0)$. Note that for every $i \in \mathbb{N}$ we can retract $M_{n}^{m}$ onto $M_{n}^{m} \cap \pi_{1}^{-1}\left(3^{-i}\right)$ simply by projecting. This leads to the well-known fact that $F$ is a Z-set in $M_{n}^{m}$. The same argument that shows that $M_{n}^{m}$ satisfies the conditions of Theorem [6 also applies to, for instance, two copies of $M_{n}^{m}$ that share an $(m-1)$ face: $M_{n}^{m} \cup\left(M_{n}^{m}+\{(1,0, \ldots, 0)\}\right)$. If $m \geq 2 n+2$, then we have that the shared face is a copy of $\mu^{n}$ as well, and that it is a Z-set in both copies of $M_{n}^{m}$. With the Z-set Unknotting Theorem [4,3.1.5] we have that if $X$ and $Y$ are spaces such that $X, Y$, and $X \cap Y$ are homeomorphic to $\mu^{n}$ and such that $X \cap Y$ is a Z-set in both $X$ and $Y$, then $X \cup Y$ is homeomorphic to $\mu^{n}$.

Theorem 7. If $n \in \mathbb{N}$, then $\operatorname{dim} \mathcal{H}\left(\mu^{n}\right)=1$.

Proof. Consider the following closed subspace of $\ell^{1}$ :

$$
\mathcal{E}=\left\{z \in \ell^{1}: 3^{i} z_{i} \in \mathbb{Z}^{+} \text {for } i \in \mathbb{Z}^{+}\right\} .
$$

According to Proposition 1 we have $\operatorname{dim} \mathcal{E} \geq 1$. Since Oversteegen and Tymchatyn [17] proved that $\operatorname{dim} \mathcal{H}\left(\mu^{n}\right) \leq 1$ it suffices to show that $\mathcal{E}$ can be imbedded in $\mathcal{H}\left(\mu^{n}\right)$. We put $m=2 n+3$ and we will work in $\mathbb{R}^{m}$. Let $u=(1,0, \ldots, 0)$ and $v=(0,1,0, \ldots, 0)$ be unit vectors in $\mathbb{R}^{m}$ and put $V=\mathbb{R} v$. We will construct a topological copy $\bar{B}$ of $\mu^{n}$ that contains the set $\{\mathbf{0}\} \cup\left\{3^{-i+1} u: i \in \mathbb{Z}^{+}\right\}$. We will then construct an imbedding $\bar{H}: \mathcal{E} \rightarrow \mathcal{H}(\bar{B})$ such that for each $z \in \mathcal{E}$ we have

$$
\bar{H}(z)(\mathbf{0})=\|z\| v \quad \text { and } \quad \bar{H}(z)\left(3^{-i+1} u\right)=3^{-i+1} u+\sum_{k=0}^{i-1} z_{k} v
$$

for every $i \in \mathbb{Z}^{+}$. Note the analogy with the two preceding sections.

Consider the unit cube $\mathbb{I}^{m}$ and its subspace $M_{n}^{m}$. We define the $m$-cells

$$
\begin{aligned}
& C=\mathbb{I}^{m}+\{u, 2 u, u+v, 2 u+v\}, \\
& D=\mathbb{I}^{m}+\{u+v, 2 u, u+2 v, 2 u+v\}
\end{aligned}
$$

and the universal Menger continua

$$
\begin{aligned}
K & =M_{n}^{m}+\{u, 2 u, u+v, 2 u+v\}, \\
L & =M_{n}^{m}+\{u+v, 2 u, u+2 v, 2 u+v\} ;
\end{aligned}
$$

see Remark 5 .

Consider the intersection $P_{1}=(K-2 v) \cap K=\pi_{2}^{-1}(0) \cap K$ and note that is a Z-set in both $K-2 v$ and $K$. Also observe that $C$ consists of two copies of $M_{n}^{m-1}$ that meet in an $(m-2)$-face that is a copy of $M_{n}^{m-2}$. By Remark 5 we have that $C$ is homeomorphic to $\mu^{n}$. Consider now $P_{2}=(L-2 v) \cap L$ and note that this intersection consists of a chain of three copies of $M_{n}^{m-1}$ whose elements meet in 
copies of $M_{n}^{m-2}$. Again by Remark [5 we have that $P_{2}$ is homeomorphic to $\mu^{n}$. It is also not hard to verify that $P_{2}$ is a Z-set in both $L-2 v$ and $L$. Note that $\pi_{1}^{-1}(3) \cap P_{1}=\pi_{1}^{-1}(3) \cap P_{2}$ is a Z-set in both $P_{1}$ and $P_{3}$ that is a copy of $M_{n}^{m-2}$. Similarly, $\pi_{1}^{-1}(1) \cap P_{1}$ and $\pi_{1}^{-1}(1) \cap P_{2}=\left(\pi_{1}^{-1}(1) \cap P_{1}\right)+v$ are copies of $M_{n}^{m-2}$ that are Z-sets in $P_{1}$, respectively, $P_{2}$. By the Z-set Unknotting Theorem there is a homeomorphism $\varphi: P_{1} \rightarrow P_{2}$ with $\varphi(x)=x$ if $x_{1}=3$ and $\varphi(x)=x+v$ if $x_{1}=1$. Observe now that $P_{1} \cup\left(P_{1}+2 v\right) \cup\left(\pi_{1}^{-1}(\{1,3\}) \cap K\right)$ is a Z-set in $K$. Similarly we have that $P_{2} \cup\left(P_{2}+2 v\right) \cup\left(\pi_{1}^{-1}(\{1,3\}) \cap L\right)$ is a Z-set in $L$. Again using the Unknotting Theorem we can find a homeomorphism $\sigma: K \rightarrow L$ such that

$$
\begin{gathered}
\sigma(x)=\varphi(x) \quad \text { for } x \in P_{1}, \\
\sigma(x+2 v)=\varphi(x)+2 v \quad \text { for } x \in P_{1}, \\
\sigma(x)=x \quad \text { for } x \in \pi_{1}^{-1}(3) \cap K, \\
\sigma(x)=x+v \quad \text { for } x \in \pi_{1}^{-1}(1) \cap K .
\end{gathered}
$$

Since both domain and range of $\sigma$ are contained in $[1,3] \times[0,3] \times \mathbb{I}^{n-1}$ we have $d(\sigma(x), x) \leq 3$ for every $x \in K$. We define the closed subsets $N$ and $F$ of $\mathbb{R}^{m}$ as follows:

$$
N=C+\{2 k v: k \in \mathbb{Z}\}=D+\{2 k v: k \in \mathbb{Z}\}=[1,3] \times \mathbb{R} \times \mathbb{I}^{n-1}
$$

and

$$
F=K+\{2 k v: k \in \mathbb{Z}\}=L+\{2 k v: k \in \mathbb{Z}\} \subset N .
$$

Note that if $x \in P_{1}=(K-2 v) \cap K$, then $\sigma(x+2 v)=\sigma(x)+2 v$. Also we have $\sigma((K-2 v) \cap K)=(L-2 v) \cap L$. These observations mean that we can extend $\sigma$ to an autohomeomorphism of $F$ by the rule $\sigma(x+2 k v)=\sigma(x)+2 k v$ for $x \in K$ and $k \in \mathbb{Z}$. Note that if $x \in F$ and $x_{1}=3$, then $\sigma(x)=x$ and that if $x_{1}=1$, then $\sigma(x)=\varphi(x)=x+v$. Note also that $F+k v=F$ for each $k \in \mathbb{Z}$.

Let $i \in \mathbb{Z}^{+}$. We define reduced copies of $L$ and $F$ :

$$
N_{i}=3^{-i} L \quad \text { and } \quad F_{i}=3^{-i} F
$$

We now take the following unions:

$$
A=\bigcup_{i=0}^{\infty} N_{i} \text { and } B=\bigcup_{i=0}^{\infty} F_{i}
$$

We denote the closures of $A$ and $B$ in $\hat{\mathbb{R}}^{m}$ by $\hat{A}$ and $\hat{B}$. Let $A_{0}=\{\mathbf{0}\} \cup\left\{x \in A: x_{2}=\right.$ $0\}$ and $B_{0}=\{\mathbf{0}\} \cup\left\{x \in B: x_{2}=0\right\}$. Observe that $\hat{A}=A \cup V \cup\left(A_{0}+\infty v\right)+\left(A_{0}-\infty v\right)$ and $\hat{B}=B \cup V \cup\left(B_{0}+\infty v\right)+\left(B_{0}-\infty v\right)$. The second statement is valid because $B$ projects onto $\left\{x \in B: x_{2}=0\right\}$ and $\left\{x \in B: x_{2}=k\right\}=\left\{x \in B: x_{2}=0\right\}+k v$ for each $k \in \mathbb{Z}$. This observation also shows that the sets $B_{0}+\infty v$ and $B_{0}-\infty v$ are Z-sets in $B^{\prime}$.

Consider now for some $i \in \mathbb{Z}^{+}$the adjacent sets $F_{i}$ and $F_{i+1}$ which meet in a subset of $\pi_{1}^{-1}\left(3^{-i}\right)$. Let $T_{i}=N_{i} \cap N_{i+1}=\left\{3^{-i}\right\} \times \mathbb{R} \times\left[0,3^{-i-1}\right]^{m-2}$. Since the line $3^{-i} u+V$ is contained in the union of the $n$-skeletons of the cubes that make up $N_{i}$ and $N_{i+1}$ we have that $F_{i} \cap T=F_{i+1} \cap T$. Recall that $F_{i+1}$ retracts by straight projection onto its "right face" $F_{i} \cap T$ and hence by induction we have that $\hat{B} \cap \pi_{1}^{-1}\left(\left[0,3^{-i}\right]\right)$ projects onto $F_{i} \cap \pi_{1}^{-1}\left(3^{-i}\right)$. This means that $\hat{\mathbb{R}} v$ is a Z-set in $\hat{B}$.

Note that $A_{0}$ is an $(m-1)$-cell and that $\hat{A}$ is homeomorphic to $A_{0} \times \hat{\mathbb{R}}$ so it is an $m$-cell. Let $k \in \mathbb{N}$ and $i \in \mathbb{Z}^{+}$and consider $M_{i}=F_{i} \cap \pi_{2}^{-1}([-k, k])$. Note that 
$M_{i} \cap T_{i}$ is a chain of $2 k$ copies of $3^{-i-1} M_{n}^{m-1}$ that meet in Z-sets that are copies of $3^{-i-1} M_{n}^{m-2}$. So by Remark $5 M_{i} \cap T_{i}$ is homeomorphic to $\mu^{n}$. By the same reasoning $M_{0}, M_{1}, \ldots, M_{i}$ is also a chain of copies of $\mu_{n}$ that meet in Z-set copies of $\mu^{n}$ and hence $\bigcup_{j=0}^{i} M_{i}$ is homeomorphic to $\mu^{n}$. So this space has the $\mathrm{DD}^{n} \mathrm{P}$ and since $i$ and $k$ are arbitrary, so does $B$. Since $\hat{B} \backslash B$ is a Z-set in $\hat{B}$ we may conclude that $\hat{B}$ has the $\mathrm{DD}^{n} \mathrm{P}$.

Let $f: \partial \mathbb{I}^{k} \rightarrow \hat{B}$ be a continuous map for $0<k \leq n$. Since $\hat{A}$ is an $m$-cell we can extend this map to a continuous $f: \mathbb{I}^{k} \rightarrow \hat{A}$. We can now use the same method as used for $M_{n}^{m}$ (see [14, §1.11]) to push the image of $f$ into $\hat{B}$. The result is that $\hat{B}$ is $\mathrm{C}^{n-1}$ and $\mathrm{LC}^{n-1}$. Since $\hat{B}$ is obviously $n$-dimensional we have that it is homeomorphic to $\mu^{n}$. Note that since $B_{0}$ is a retract of $\hat{B}$ it is also $\mathrm{C}^{n-1}$.

We let $\bar{A}$ and $\bar{B}$ be the closures of $A$, respectively $B$, in $Q^{m}$. Note that $\bar{A}=$ $A \cup V \cup\{\alpha, \beta\}$ and $\bar{B}=B \cup V \cup\{\alpha, \beta\}$. We can obtain $\bar{B}$ from $\hat{B}$ by identifying the "faces" $B_{0}+\infty v$ and $B_{0}-\infty v$ to points $\alpha$ and $\beta$. Since these faces are Z-sets that are $C^{n-1}$, we have, according to the Z-set Shrinking Theorem [4, 4.1.2], that $\bar{B}$ is homeomorphic to $\mu^{n}$.

The remainder of the proof is completely analogous to the argument in $\S 5$ : we only need to replace every 2 by a 3 . We finish by stating the resulting definition of the imbedding $\bar{H}: \mathcal{E} \rightarrow \mathcal{H}(\bar{B})$ for the record: if $z \in \mathcal{E}$, then

$$
\bar{H}(z)(x)= \begin{cases}3^{-i} \sigma^{j}\left(3^{i} x\right)+\sum_{k=0}^{i-1} z_{k} v, & \text { if } x \in F_{i} \text { with } j=3^{i} z_{i} \\ x+\|z\| v, & \text { if } x \in V \\ \alpha, & \text { if } x=\alpha \\ \beta, & \text { if } x=\beta\end{cases}
$$

Remark 6. Again we have that $\mathcal{E}$ is a copy of complete Erdős space and that $\bar{H}$ is a semigroup homomorphism.

Remark 7. Again we note a number of properties of the imbedding $\bar{H}$ that will be needed in [12. The reasoning is analogous to Remark 3. For fixed $x \in \bar{B} \backslash V$ we have that $\bar{H}(z)(x)$ depends on only finitely many coordinates of $z \in \mathcal{E}$. Let $Z$ stand for $\mathcal{E}$ in the preceding proof, but equipped with the zero-dimensional topology that $Z$ inherits from the product space $\mathbb{R}^{\mathbb{Z}^{+}}$, and let $\psi: \mathcal{H}(\bar{B}) \rightarrow \bar{B}^{A}$ be defined by the rule $\psi(h)=h \uparrow A$, where $A=\left\{3^{-i+1} u: i \in \mathbb{Z}^{+}\right\} \cup\{\alpha\}$. We have that both $\psi \circ \bar{H}: Z \rightarrow \psi(\mathcal{H}(\bar{B}))$ and $\bar{H}: \mathcal{E} \rightarrow \mathcal{H}(\bar{B})$ are closed imbeddings.

Remark 8. If $O$ is a nonempty open subset of $\mu^{n}$, then $\mathcal{H}\left(\mu^{n}\lceil O)\right.$ is also onedimensional. Again, this result is needed in [13. Consider the cube $T=\{3\} \times \mathbb{I}^{m-1}$. Note that $M=T \cap \bar{B}$ is a copy of $M_{n}^{m-1}$ (and of $\mu^{n}$ ) and a Z-set in $\bar{B}$. Since for every $z \in \mathcal{E}, \bar{H}(z)$ fixes every point $x$ with $x_{1}=3$, we have that $\operatorname{dim} \mathcal{H}(\bar{B}\lceil\bar{B} \backslash M)=1$. Because of the fractal nature of $\mu^{n}$ we can find a subset $P$ of $O$ whose boundary $Z$ is a Z-set in $P$ and with the property that both $P$ and $Z$ are copies of $\mu^{n}$. Then there is a homeomorphism $h: \bar{B} \rightarrow P$ with $h(M)=Z$. Thus $\mathcal{H}(P\lceil P \backslash Z)$ contains a copy of $\mathcal{E}$ and is obviously identical to $\mathcal{H}\left(\mu^{n}\lceil P \backslash Z)\right.$, which in turn is contained in $\mathcal{H}\left(M_{n}^{n+1}\lceil O)\right.$.

By the same argument as in Remark 4 we also find that every subset of $\mathcal{H}\left(\mu^{n}\right)$ with a nonempty interior contains a closed copy of every complete almost zerodimensional space. 
We finish with some questions. A natural question is of course whether Claim 1 is valid. Perhaps more interesting is the question whether there exist nontrivial homogeneous (Peano) continua with a zero-dimensional homeomorphism group; cf. Brechner [6].

\section{REFERENCES}

[1] R. D. Anderson, A characterization of the universal curve and a proof of its homogeneity, Ann. of Math. 67 (1958), 313-324. MR0096180|(20:2675)

[2] R. D. Anderson, One-dimensional continuous curves and a homogeneity theorem, Ann. of Math. 68 (1958), 1-16. MR0096181 (20:2676)

[3] R. Arens, Topologies for homeomorphism groups, Amer. J. Math. 68 (1946), 593-610. MR 0019916 (8:479i)

[4] M. Bestvina, Characterizing k-dimensional universal Menger compacta, Mem. Amer. Math. Soc. 71 (1988), no. 380, vi+110. MR0920964 (89g:54083)

[5] B. L. Brechner, On the dimensions of certain spaces of homeomorphisms, Trans. Amer. Math. Soc. 121 (1966), 516-548. MR0187208 (32:4662)

[6] B. L. Brechner, Questions on homeomorphism groups of chainable and homogeneous continua, Topology Proc. 7 (1982), 391-393.

[7] J. W. Cannon, A positional characterization of the $(n-1)$-dimensional Sierpinski curve in $S^{n}(n \neq 4)$, Fund. Math. 79 (1973), no. 2, 107-112. MR0319203 (47:7748)

[8] J. J. Dijkstra, On homeomorphism groups and the compact-open topology, Amer. Math. Monthly, to appear.

[9] J. J. Dijkstra, A criterion for Erdös spaces, Proc. Edinburgh Math. Soc., to appear.

[10] J. J. Dijkstra and J. van Mill, Homeomorphism groups of manifolds and Erdös space, Electron. Res. Announc. Amer. Math. Soc. 10 (2004), 29-38. MR2048429

[11] J. J. Dijkstra and J. van Mill, On the group of homeomorphisms of the real line that map the pseudoboundary onto itself, preprint.

[12] J. J. Dijkstra and J. van Mill, Erdös space and homeomorphism groups of manifolds, preprint.

[13] J. J. Dijkstra, J. van Mill, and J. Steprāns, Complete Erdös space is unstable, Math. Proc. Cambridge Philos. Soc. 137 (2004), 465-473. MR2092071

[14] R. Engelking, Dimension Theory, North-Holland, Amsterdam, 1978. MR0482697(58:2753b)

[15] P. Erdős, The dimension of the rational points in Hilbert space, Ann. of Math. 41 (1940), 734-736. MR0003191 (2:178a)

[16] K. Kawamura, L. G. Oversteegen, and E. D. Tymchatyn, On homogeneous totally disconnected 1-dimensional spaces, Fund. Math. 150 (1996), 97-112. MR.1391294 (97d:54060)

[17] L. G. Oversteegen and E. D. Tymchatyn, On the dimension of certain totally disconnected spaces, Proc. Amer. Math. Soc. 122 (1994), 885-891. MF,1273515 (95b:54040)

[18] G. T. Whyburn, Topological characterization of the Sierpinski curve, Fund. Math. 45 (1958), 320-324. MR0099638 (20:6077)

Faculteit der Exacte Wetenschappen / Afdeling Wiskunde, Vrije Universiteit, De Boelelaan 1081A, 1081 HV Amsterdam, The Netherlands

E-mail address: dijkstra@cs.vu.nl 